\title{
Safeguarded Teleoperation for Lunar Rovers
}

Eric Krotkov, Reid Simmons, Fabio Cozman, and Sven Koenig Camegie Mellon Univ. 
The appearance of the ISSN code at the bottom of this page indicates SAE's consent that copies of the paper may be made for personal or internal use of specific clients. This consent is given on the condition however, that the copier pay a $\$ 7.00$ per article copy fee through the Copyright Clearance Center, Inc. Operations Center, 222 Rosewood Drive, Danvers, MA 01923 for copying beyond that permitted by Sections $107 \propto 108$ of U.S. Copyright Law. Thisconsent does not extend to other kinds of copying such as copying for general distribution, for advertising or promotional purposes, forcreating new collective works, or for resale.

SAE routinely stocks printed papers for a period of three years following date of publication. Direct your orders to SAE Customer Sales and Satisfaction Department.

Quantity reprint rates can be obtained from the Customer Sales and Satisfaction Department.

To request permission to reprint a technical paper or permission to use copyrighted SAE publications in other works. contact the SAE Publications Group.

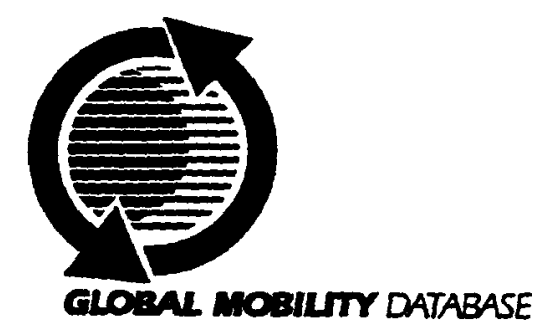

Al SAE papers, standards, and solected books are abstracted and indexed in the Glabal Mobility Database.

No part of this publication maybe reproduced in any form, in an electronic retrieval system or otherwise, without the prior written permission ofthepublisher.

\section{ISSN 0148-7191}

Copyright 1996 Society of Automotive Engineers, inc.

Positions and opinions advanced in this paper are those of the author(s) and not necessarily those of SAE. The author is solety responsible for the content of the paper. A process is available by which discussions will be printed with the paper if it is published in SAE Transactions. For permission to publish Ihiipaper in full or in part,contact the SAE Publications Group.

Persons wishing to submit papers to be considered for presentation or publication through SAE should send the manuscript or a 300 word abstract of a proposed manuscriptto: Secretary, Engineering Meetings Board, SAE. 


\title{
Safeguarded Teleoperation for Lunar Rovers
}

\author{
Eric Krotkov, Reid Simmons, Fabio Cozman, and Sven Koenjig
}

\begin{abstract}
In this paper we present recent advances in developing and validating the safeguarded teleoperation approach to timedelayed remote driving. This approach shares control of the rover using a command fusion strategy: In benign situations, users remotely drive the rover; in hazardous situations, a safeguarding system running on-board the rover overwrites user commands to ensure vehicle safety. This strategy satisfies users, because it allows them to drive (except in hazardous situations), while maintaining the integrity of the rover and mission.

We present results from experiments on untrained teleoperators with and without safeguarding, which reveal needs to be met by future user interfaces. We describe three technical advances in safeguarding: improving the accuracy of dead reckoning by a factor of 2 , speeding up the controller by a factor of 18, and developing an area-based rather than a path-based obstacle avoidance planner in order to circumvent map merging problems. Finally, we discuss a field trial validating the approach in a $10 \mathrm{~km}$ traverse, demonstrating the effectiveness of safeguarding, even with malicious drivers.
\end{abstract}

\section{Introduction}

In our previous work on Mars rovers [10], we aimed for a high degree of autonomy, because teleoperation with long (up to 45 . minute) time delays severely limits rover capabilities. In our current work on lunar rovers, we aim for a mix of teleopera. tion and autonomous safeguarding, because the Moon is close enough that teleoperation is possible, yet far enough away that time delays prove troublesome.

Our work belongs to a larger Lunar Rover Initiative, which aims to conduct a lunar mission sponsored not by government agencies, but by private ventures dedicated to public participation in space exploration [8]. A central goal of the initiative is to enable untrained people to experience, and at times directly control, a rover visiting historic sites and unexplored areas on the Moon.

This goal is ambitious, because remotely driving a lunar rover presents a number of technical challenges, with time-delay foremost among them. The largest distance between Earth and the Moon is 407,000 kilometers, leading to a communication delay of about 2.5 seconds in each direction (1.4 seconds between Earth and the Moon, and an estimated 1.1 second communication delay on Earth). Thus, if the rover is aboutto hit an obstacle on the Moon, the teleoperator on Earth notices this situation 2.5 seconds later, and (assuming instant response) their evasive motion commands reach the rover another 2.5 seconds after that five seconds after hitting the obstacle, potentially crippling the rover and ending the mission.

A classical approach to timedelayed teleoperation requires the user to spocify woypoints which the ground station uplinks to the rover, which drives itself to them. Althougheffective, this approach does not meet the needs of untrained users - whether they are astomens d entertainmentparks, visitors at museums, students at school, or family members in living rooms-who require intuitiveinterfaces that permit them to keep their hands on the "steering wheel" as much as possible. These users need a degree of control greater than clicking waypoints on a screen.

Responding to these needs, we have developed the safeguarded teleoperation approach, which shares control of the rover using a command fusion strategy. In benign situations, users remotely drive the rover. In hazardous situations, a safeguarding system running on-board the rover overwrites user commands to ensure vehicle safety. This strategy satisfies users, because it allows them to drive (except in hazardous situations), while maintaining the integrity of the rover and mission.

While oprating in safeguarding mode, the rover must exhibit many of the characteristics of autonomy: perception and understanding of its surroundings, monitoring its own status and health, knowing where it is and where it is going, and taking appropriateactions to maintain its own safety. We observe that the safeguarding system must be on-board in order to react to hazards without communication delay.

In previous work, we implemented a complete safeguarded teleoperation system and validated it by traversing one kilometer of lunar-like terrain $(9 ; 23 ; 221$. The mobile base for the traverse was Ratler (Figure 1), developed by Sandia National Laboratory. This paper addresses a simplified version (Figure 2) of the complete system. The key simplification is to omit goal achievement modules, which in the complete system were implemented as a route plannerand a position and heading goalseeking module. The simplification permits us to concentrate directly on the interaction between stereo-based safeguarding and user control.

This work makes two key contributions. First, it has begun studying the effects of safeguarding on untrained operators in remote driving tasks. The methodology and results of such studies may have profound consequences for all forms of timedelayed teleoperation. Second, it has achieved an unprecedented ten-kilometer traverse under safeguarded teleoperation control, which was enabled by advances in dead reckoning, task syn- 


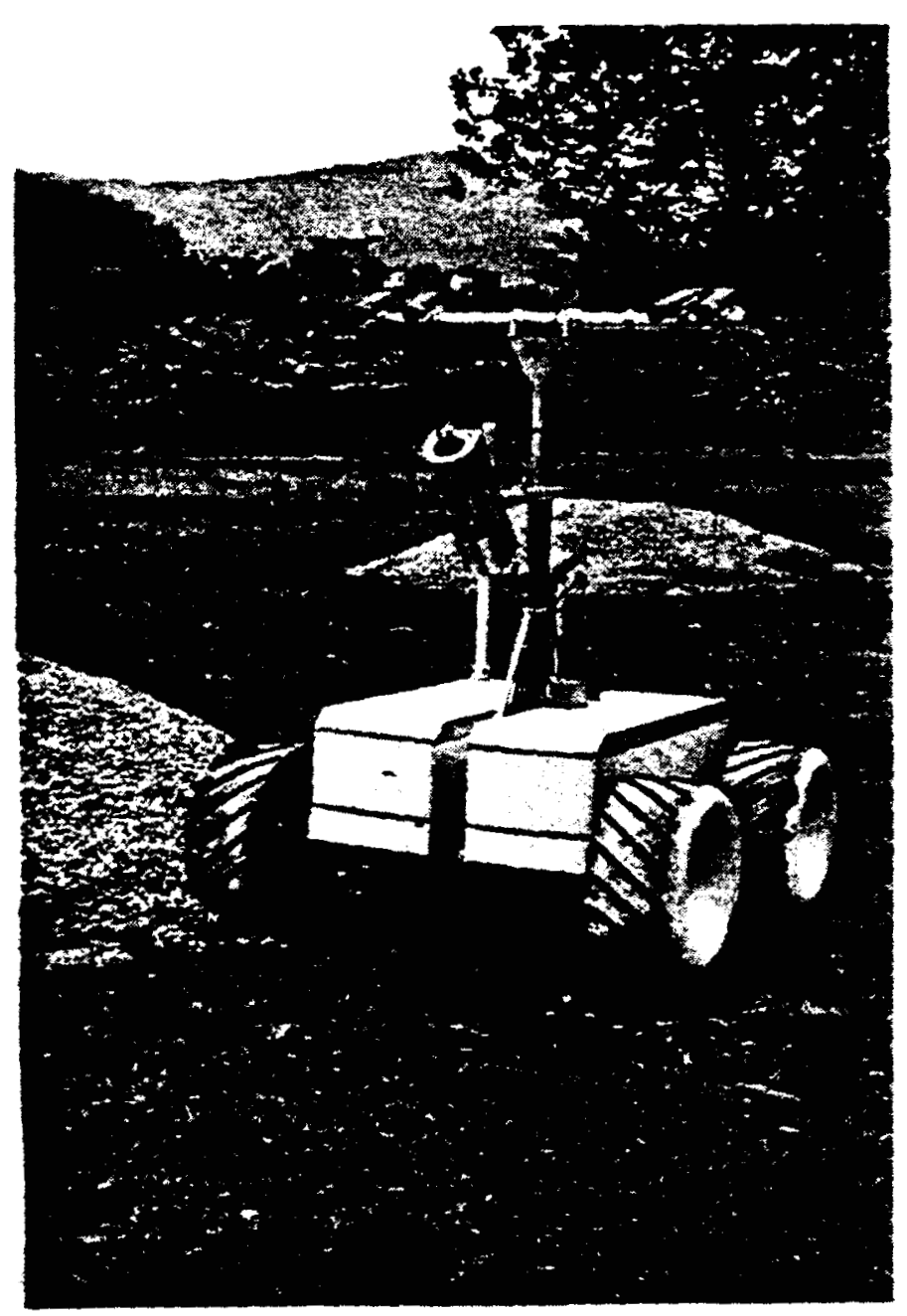

Figure 1: Ratler mobile robot

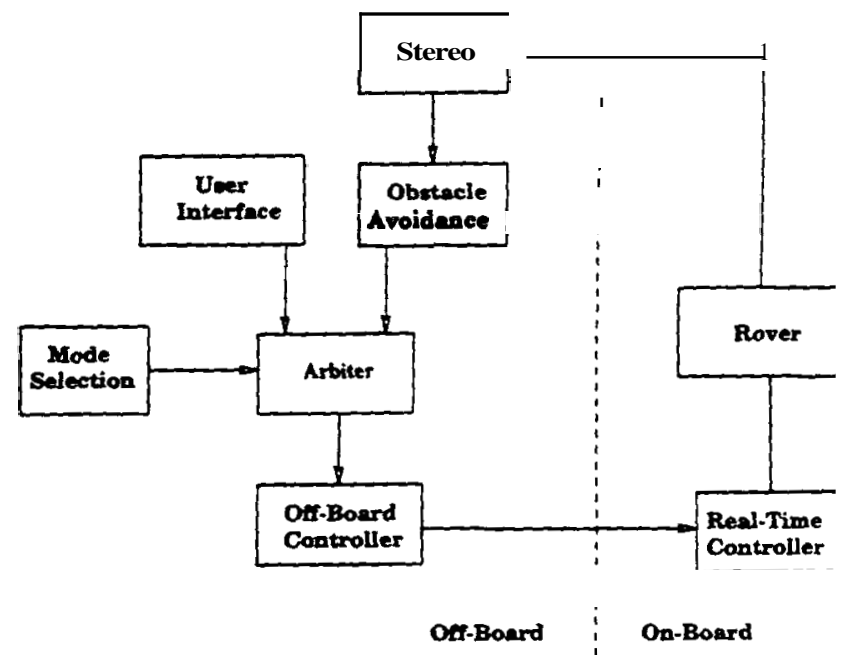

Figure 2: Safeguarded teleoperation system architecture

chronization, and stereo-based obstacle avoidance planning.

The paper is organized as follows. In the two next sections, we survey related literature and present a study of human performance in time-delayed remote driving tasks, both with and without safeguarding. In the two following sections, we describe key technical advances over our previous efforts, including improvements in dead reckoning and real-time control, and an innovative area-based approach to obstacle avoidance planning. In the final two sections, we present the results of field trials, and discuss key issues and future work.

\section{Related Work}

A number of researchers have addressed the problem of stereobased remote driving under planetary constraints. Some achieve near real-time performance by virtue of special-purpose hardware, such as Datacube systems [14], digital signal processors [19], and transputer arrays 11; 181. Unlike these approaches, we have restricted our attention to genaal-purpose processors (in this case, a Sparc 10), with computing power comparable to flightqualifiable computers such as the $R 4400$ and $R 4600$.

Other researchers have demonstrated stereo-based remote driving using modest computing resources $[17 ; 111$. [2]. The distinction of our approach is that it has been validated by more thorough testing.

Several recent efforts have direct relevance to lunar robotics, including construction of lunar bases [3], and lunar exploration (Pele at Kiluaea, unpublished). Other recent efforts have developed related planetary rover technology, some intended for specific future missions [15], and some intended for planetary exploration in general [4]. None of these efforts competes directly with the stereo-basad safeguarding approach.

The only unmanned rovers to drive on the lunar surface were teleoperated by the Soviets in the 1960s [16]. Teleoperators reported that remote driving was adifficult and exhausting task. The next rover to drive on a planetary surface will be the Mars Pathfinder Mictorover Flight Experiment [20], which will use the waypoint designation approach discussed earlier. 
Although the "safeguarded teleoperation"terminology is new, the fundamental concepts of shared and traded control, on which the approach is based, are well-known in remote manipulator control. Similar approaches have been implemented in several mobile robot efforts, including CARD [12] and STRIPE [6]. With the former, the distinction of our approach is in allowing continuous user input rather than discrete waypoints. With the latter, the distinction is in using passive stereo rather than active rangefinders for acquiring three-dimensional information.

In summary, there is an active and growing community developing lunar-relevant approaches for remote driving. Ore stereo-based safeguarded teleoperation approach is unique in enabling users to share continuous control of the rover, and in the maturity of the ten-kilometer validation.

\section{Teleoperation With and Without Safeguarding}

We performed a series of experiments to evaluate the effect of the command fusion strategy on the behavior of untrained rover operators. Our hypothesis was that the presence of safeguarding software would decrease the fatigue and frustration of the subjects and, as a result, increase their overall performance and enjoyment. Since the experimentsperformed to test this hypothesis measured human factors and not software quality, a social scientist validated them. In the following, we describe the experiments and the impact of their results on the development of arr user interface for timedelayed teleoperation.

\subsection{Experiments}

Our experimental setup simulated the time-delay between Farth and the Moon. The user was placed in a control room, and provided with undelayed real-time video from the camera onboard the rover. They used a minimal, joystick-like interface to issue a strean of commands that controlled the speed and curvature (rate of turn) of the rover. These commands were then delayed by five seconds, twice the communication delay between Eath and the Moon. Since our safeguarding software was not fast enough yet. its function was performed by a person from our research group, in the following called the "wizard." The wizard was in line of sight with the rover and was provided with the user command that was about to be executed by the rover. They could either allow the rover to execute the user command, send a stop command instead (called "vetoing"the user command), or slightly alter the user command using their own joystick-like interface. The commands of the wizard were not subject to any time-delay. The interface of the user indicated whether the wizard had vetoed the current command.

We tested our command fusion strategy on eleven subjects, mostly undergraduate and graduate students. Their initial training was minimal: they were given only two two-minute test drives on a small training course, one test drive without timedelay and one with a five-second timedelay. The subjects were then instructed to drive as quickly as possible through an obstacle course to a brightly marked finish line that was visible from the start position of the rover. The obstacle course was $\mathbf{5 4}$ meters long and contained 32 obstacles, mostly old tires. The obstacle spacing varied from 3 to $\mathbf{4} \mathbf{5}$ meters.

We compared trials with no wizard (N) and with a wizard that vetos shortly before the rover would hit an obstacle (V). This control strategyhas the disadvantagethat, when the wizard vetos a user command, the user can react to it only with a time-delay

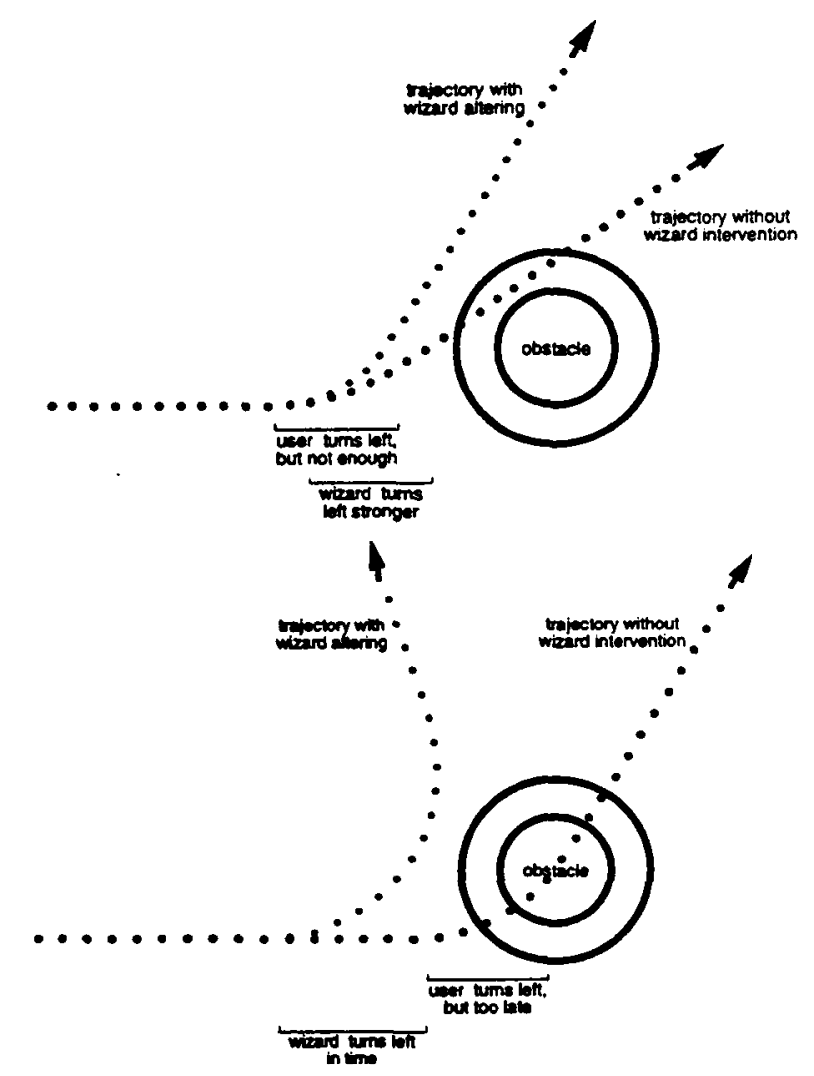

Figure 3: Successful intervention (top) and oversteering

of five seconds. Thus, after a veto command, the rover usually sits idle for this period of time, which is a potential cause of frustration. We therefore also tested a control strategy in which the wizard alters the user commands slightly to avoid collisions with obstacles and vetos only when absolutely necessary (A). Each subject, knowing the current control strategy, performed two trials with two different control strategies of the wizard.

Even if the wizard did not intervene, all subjects but one were able to complete the obstacle course successfully and hit only approximately one obstacle per trial. However, nine of the eleven subjects reported the the hardest aspect of their driving experience was the time-delay. After each trial, they rated their fatigue (Fat), frustration (Fru), and overall enjoyment (Enj) on a scale from 1 (lowest) to 10 (highest).

Table 1 tabulates the results. Given the small samplesize, we expected the data to be informative, but not definitive.

We expected to observe a tendency for the presence of the wizard to decrease the fatigue and frustration of the subjects and, as a result, increase their overall enjoyment and performance. Unexpectedly, this did not happen. For example, there were nine subjects who had one trial where the wizard vetoed $(\mathrm{V})$. They rated their fatigue as $\mathbf{3 . 3}$ on average. The subjects during whose other trial the wizard did not intervene at all $(\mathrm{N})$ rated their fatigue in this other trial $\mathbf{0 . 3}$ points higher, for an average score of 3.6.

Post-driving interviews revealed two reasons for this unexpected finding. First, many subjects did not take advantage of the presence of the wizard. Eight subjects did not adapt their 


\begin{tabular}{|c|c|c|c|c|c|c|c|c|c|c|c|c|c|c|}
\hline \multirow{4}{*}{$\begin{array}{l}\text { strategy } \\
\text { of wizard }\end{array}$} & \multirow[t]{4}{*}{ runs } & & \multirow{3}{*}{\multicolumn{3}{|c|}{ score of stralegy }} & \multicolumn{9}{|c|}{ compared to straxegy of wizard } \\
\hline & & & & & & \multicolumn{9}{|c|}{ change in score } \\
\hline & & & & & & \multicolumn{3}{|c|}{ Dointervention (N) } & \multicolumn{3}{|c|}{ wizard vetos $(\nabla)$} & \multicolumn{3}{|c|}{ wizard aters $(\bar{A})$} \\
\hline & & & Fat & F̃̃ & Enj & Fat & F & Eoj & Faz & FTy & Enj & Fat & Fis & Enj \\
\hline $\bar{N}$ & 5 & $\begin{array}{l}\text { mean } \\
\text { variance }\end{array}$ & $\begin{array}{l}3.0 \\
2.0\end{array}$ & $\begin{array}{l}2.6 \\
1.0\end{array}$ & $\begin{array}{l}6.4 \\
1.8\end{array}$ & & & & $\begin{array}{r}-0.3 \\
0.9\end{array}$ & $\begin{array}{r}+0.0 \\
0.7\end{array}$ & $\begin{array}{r}-1.0 \\
0.7\end{array}$ & $\begin{array}{l}0.5 \\
0.3\end{array}$ & $\begin{array}{r}+1.0 \\
0.0\end{array}$ & $\begin{array}{r}+0.0 \\
0.0\end{array}$ \\
\hline $\bar{v}$ & 9 & $\begin{array}{l}\text { mean } \\
\text { vatiance }\end{array}$ & $\begin{array}{l}3.3 \\
5.1 \\
\end{array}$ & $\begin{array}{l}2.9 \\
1.8\end{array}$ & $\begin{array}{l}6.1 \\
4.1\end{array}$ & $\begin{array}{r}+0.3 \\
0.9\end{array}$ & $\begin{array}{l}+0 . \\
0.7\end{array}$ & $\begin{array}{r}+1.0 \\
0.7\end{array}$ & & & & $\begin{array}{r}-0.3 \\
3.0\end{array}$ & $\begin{array}{r}+0.5 \\
1.8\end{array}$ & $\begin{array}{r}-0.8 \\
0.8\end{array}$ \\
\hline $\bar{A}$ & 8 & $\begin{array}{l}\text { mean } \\
\text { variance }\end{array}$ & $\begin{array}{l}2.8 \\
1.9\end{array}$ & $\begin{array}{l}3.4 \\
4.0\end{array}$ & $\begin{array}{l}6.0 \\
3.6\end{array}$ & $\begin{array}{r}+0.5 \\
0.3\end{array}$ & $\begin{array}{r}-1 . \\
0 . C\end{array}$ & $\begin{array}{r}+0.0 \\
0.0\end{array}$ & $\begin{array}{r}+0.3 \\
3.0\end{array}$ & $\begin{array}{r}-0.5 \\
1.8\end{array}$ & $\begin{array}{r}+0.8 \\
0.8\end{array}$ & & & \\
\hline
\end{tabular}

Table 1: Teleoperation trial results

driving strategy to the strategy of the wizard. They wanted to be in control of their driving and did not rely at all on the presence of the wizard, for example by increasing the speed of the rover. (There was one exception to this rule, backing up, since there was no camera on the rover pointing backwards.) Instead, the subjects always drove slowly through the course in order to avoid obstacles. Only three subjects changed their driving strategy based on the strategy of the wizard. One subject decided to let the wizard protect the robot from obstacles, the other two became more cautious when a wizard was present. They viewed an interventionby the wizard as their failure. This cautious behavior might have been caused by the maturity of the subjects or the known presence of a human wizard (as opposed to an automaticsafeguarding system). Some subjectseven tried to run the rover into the wizard when the wizard was in the field of the camera view. We expect that the result would have been differenthad we used high-school instead of university students or had we not told the subjects that the safeguarding function was performed by a person.

Second, the altering strategy of the wizard can cause oversteering. When the wizard altered the user commands, some subjectsreported that the rover became more difficultto control, althoughno subject was able to detect when their commands had been altered. Figure 3 describes two situations that we observed during the tests, contrasting a successful wizard intervention with one leading to oversteering. Figure 3 (left) depicts a situation in which the intervention of the wizard achieved its intended effect. This is the case when the wizard slightly corrects either the curvature of the user command or the length of the turn. Figure 3 (right) shows a situation in which the intervention of the wizard did not achieve its intended effect. In this case, the user turned too late, long after the wizard started to tum. When the user finally turned, the front of the rover was clear and consequently the wizard passed through the user's tum commands. As a consequence, the rover turned for an extended period of time and oversteered.

\subsection{Towards a New User Interface}

During the experiments, we made the following observations that are proving useful for developing a more sophisticated user interface:

All subjects used short-term control strategies to avoid obstacles and make progress towards the goal line, no one used a global path planning strategy. Consequently, their paths were often highly inefficient: there were only two trials in which subjects followed a shortest path to the goal line.

Given the visually rich environment and the wide field of view provided by the camera on the rover. maintaining heading towards the goal line was a problem for only one subject.
However, if an obstacle left the field of view, then its presence was no longer remembered by the subjects. This proved to be a problem, because there was a blind spot of $64 \mathrm{~cm}$ directly in front of the rover in which obstacles could not be seen. Once an obstacle entered this blind spot, the subjects forgot about it. They were then surprised when the wizard vetoed their forward commands soon afterwards. Three subjects therefore explicitly asked us to either eliminate the blind spot or provide a global map that shows the rover and the obstacles that surround it.

Most subjects maintained the maximum speed of the rover in the middle of the offered velocity range, even if the largest velocity allowed was drastically reduced from $42 \mathrm{~cm} / \mathrm{sec}$ to 21 $\mathrm{cm} / \mathrm{sec}$. We attribute this behavior to the limited training time; half of the maximum speed appears to be a choice that the user, intuitively, acoepts as safe and not too bold, given that they do not have the experienceyet to make their own judgements.

Three subjects, two of whom were completelyunfamiliar with graphical user interfaces, initially had problems understanding the function of the input device. They believed that pointing into the eastern region of the "clock face" would automatically turn theroveruntil it faced cast. These subjects would have preferred either an actual joystick or a steering wheel. Two subjects did not like that the mouse button had to be pressed continuously to make the rover move ("gas pedal control"). They would have preferred an active stop command (" "brake pedal control"). Two subjects mentioned that it was hard to watch two monitors, the one that provided the video from the rover and the one that displayed the input device.

Our conclusion from these observations is that more sophisticated approaches to safeguarded teleoperation are needed. We have begun work on a new user interface with the following properties:

First, the user is given more control over the safeguarding software. Although the safeguarding software always stops the rover before it hits an obstacle, the user is able to decide when the system is allowed to alter their commands and when it is allowed to take over control completely, for example to get the rover out of a tricky situation. We expect the user to rely more on the safeguarding mechanisms when he feels more in control of them.

Second, the user uses a steering wheel to control the rover, together with gas and brake pedals - input devices that most users can be expected to be familiar with. The speed control is non-linear, with smallerspeds given a larger range of the input.

Third, to reduce the cognitive load, we provide users with more information. We eliminate the blind spot in front of the rover and provide the user with a predictive display that, based on the commands of the user, estimates the future rover position and thus predicts for them the future movement of the rover. 


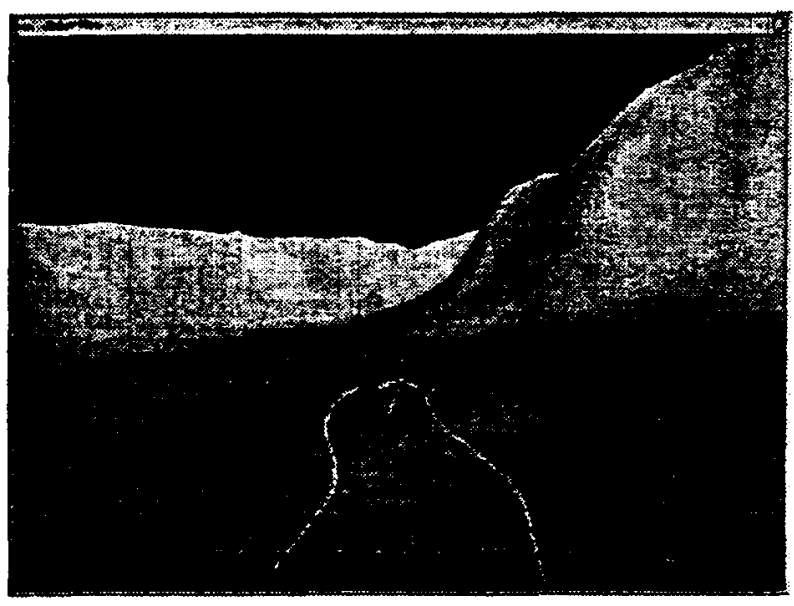

Figure 4: Predictivedisplay

The advantage is that the user need not predict the situation in which their current command will apply; this situation is now predicted and visualized by the user interface. We are also considering installing a second camera on the rover that points backwards and whether to let the user interface display a local map of known obstacles around the rover, using an elevation map created by the on-board stereo perception system and then transmitted to the user control station.

Although the new user interface is still under development, we [ 131 have simulated the predictivedisplay (Figure 4), which consists of twolines, projected onto the video display, that trace the predicted path of the rover in the next five seconds. It also shows an arrow that describes the position and heading of the rover in five seconds. Initial experiments with this predictive display have been encouraging: users produced more continuous command streams, drove faster, oversteered less, and hit fewer obstacles when no wizard was present.

\section{Real-Time Controller}

In this section we discuss two efforts to enhance the real-time controller. The first improves the accuracy of dead reckoning by a factor of $\mathbf{2}$. The second implements feedback control that increases response time by a factor of 18 . These enhancements contributed substantially to the success of the field trials.

\subsection{Dead Reckoning}

Eight different Sensor measurements are available inside Ratler: four encoders (one for each wheel), three inclinometers (roll, left pitch, and right pitch), and a compass. We have presented one approach to using these measurements for dead reckoning [9]. We have also presented an alternative approach using an inertial measurement unit [5]. In this section, we present an evolution of the first approach, and the results of a rigorous test program.

The signals from the three inclinometers are quite stable and noiseless; on the other hand, the compass signal presents high levels of noise during motion. Further tests with the compass and similar devices from other robots available in our group pointed to the same general conclusion: the existence of large metallic structures causes sudden and unexpected changes in orientation measurements. Since we conducted our tests in the vicinity of large buildings, we were forced to abandon the compass altogether, and rely solely on encoder and inclinometer integration.

Take $(x, y, z)$ for position, $\theta$ for yaw (orientation)and $\phi$ for average pitch from the filtered inclinometer signals. We computed the coordinates with respect to a global coordinate system:

$$
\begin{aligned}
& x_{n+1}=x_{n}+\frac{e_{r}+e_{l}}{2} \cos \theta_{n} \cos \phi \\
& y_{n+1}=y_{n}+\frac{e_{r}+e_{l}}{2} \sin \theta_{n} \sin \phi \\
& z_{n+1}=z_{n}+\frac{e_{r}+e_{l}}{2} \sin \phi \\
& \theta_{n+1}=\frac{e_{r}-e_{l}}{B},
\end{aligned}
$$

where $\mathrm{B}$ is the wheelbase and $\boldsymbol{e}$, and $\boldsymbol{e}_{\boldsymbol{l}}$ are respectively the encoder displacements expressed in metric units.

To quantify the performance of this approach, we built a test site in a $10 \times \mathbf{2 0}$ meter area with rough but uniform terrain, in which we drew a grid with intervals of $\mathbf{0 . 2 5}$ meter. We attached a piece of chalk to the center of Ratler, so that motion of the robot produced a trail of chalk on the ground.

We commanded Ratler to follow $\mathbf{1 0}$ different arcs, varying from a sharp right turn (radius of 3 meters) to a sharp left turn (same radius). We repeated each arc 3 times. We repeated straight motions approximately 15 times. Misalignment of one of the wheels to the right by a mere $2^{\circ}$ caused the robot to drift 0.6 meter every 20 meters (3\% error). Skid steering contributed an additional 0.75 meter drift (4\% error), on average, for commanded linear trajectories. We compensated these errors by introducing correction factors in the encoder readings. This substantially reduced the mean error. For straight lines, average m or fell to 0.3 meter for every 20 meter trajectory $(1.5 \%$ error $)$. However, for abrupt turns performance is poor; for instance, a 3 meter radius turn leads to $10 \%$ mean error. This poor performance during $\mathrm{m} s$ appears to be an inevitable consequence of the slippage caused by skid steering.

\section{Task Synchronization}

In an attempt to achieve modularization, the original design for the low-levelcontrol systemdivides dead reckoning and control into five tasks, all executing concurrently and sharing memory. The task responsible for reading the encoders executes every IO $\mathrm{ms}$ and the compass-reading task executes every $100 \mathrm{~ms}$. The task responsible for wheel speed control executes every $100 \mathrm{~ms}$, and occurs asynchronously with respect to all other tasks. The original design does not includefeedback control for the wheels. During tests reported in [9], it became obvious that closed-loop control was necessary.

The modular architecture, in which different tasks behave independently,creates artificial sources of noise. In somecycles, the 100 ms-time-based low-level control task obtains the result of ten readings from the $10 \mathrm{~ms}$-time-based encoder task; in other cycles, it obtains nine or eleven readings, due to minor delays caused by task swapping. As a result, the integration of encoder signals received values corrupted by $\pm 10 \%$. Figure 5 illustrates this phenomenon by graphing measured speed against time in a long run (225 seconds). Speed gradually increased and then gradually decreased. Notice that noise is not additive; instead, it depends on speed, because the lack of synchronizationinduces speed-relativeerrorsrather than absolute errors. With such noisy 


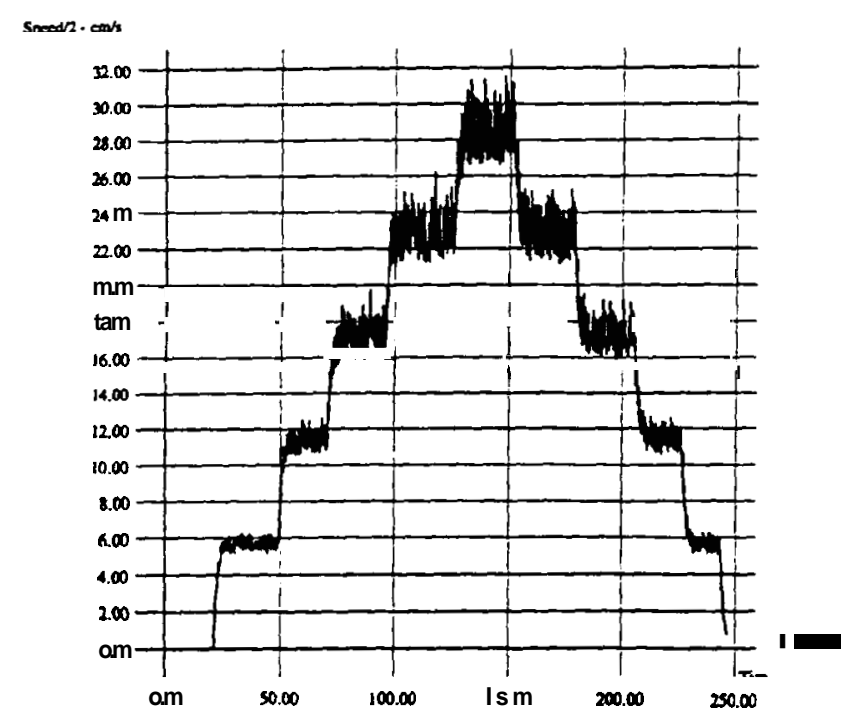

Figure 5: Speed-dependent noise in modular design

signals, feedback control tends to lead the system into unstable behavior, causing the rover to shake, fight itself, and behave in schizophrenicbursts. The best possible gains are stable only for time constants of 12 seconds, meaning that the robot would take approximately 30 seconds to obey an arc command.

We redesigned the system to synchronize the critical tasks of data acquisition, dead reckoning, and control. This removes the independence among functional units, but improves performance dramatically, suppressing noise significantly, and reducing time constants to 0.67 second ( 18 times faster response). We now describe the controller tuning.

Tests with the four wheel motors indicated that their parameters were virtually identical; the electrical constants were practically negligible. The motors were analyzed with various load conditions: Ratler suspended, Ratler on the ground, Ratler loaded with stones. In all cases, there was little variation in the mechanical constant of the motors. This was due to the reduction gears $(100: 1)$ coupled to the motors, which consume a substantial portion of the power produced by the motors, irrespective of load. Thus, we used a first-order model for the motors:

$$
\dot{v}=\frac{K}{T} v-\frac{1}{T} u
$$

where $u$ is the input, $v$ is the measured speed, $K$ is the motor's steady state gain, and Tis the motor's mechanical time constant. The time constant of all motors, with full load was determined to be $\mathbf{T}=\mathbf{0 . 1 5}$ second. The steady-stategain was more complicated; the motors had a dead zone from 0.0 to $0.03 \mathrm{~m} / \mathrm{s}$, and then exhibited highly linear behavior until speeds of $0.91 \mathrm{~m} / \mathrm{s}$. The gain $K=1.248$ reasonably approximates this behavior for all commanded speeds. We implemented a proportional controller, with constant gain set to unity. This gain fixed the dominant pole of the motors at (approximately) -1.5, guaranteeing fast response $(0.67$ second time constant).

\section{Obstacle Avoidance Planning}

A key to our approach is that the rover should, in real-time, evaluate where it is safe for it to be heading, based on local sensing (e.g., stereo vision). These evaluations can then be used either to drive the rover autonomously, or to safeguard the rover by over-riding (or even by modifying) user commands (Section 3).

In our previously reported experiments with Ratler $[9 ; 21$; 221, we adapted a local obstacle avoidance planner called Ranger, which was developed at CMU for ARPA's Unmanned Ground Vehicle (UGV) program for cross-country navigation [7]. Ranger works by analyzing the paths the vehicle would traverse along the terrain for a number of different steering angles, and choosing the one that evaluates as the safest.

Terrain data for Ranger is obtained from stereo vision. To make the stereo computation tractable, only a small segment of the stereo image (about 2\%) is requested, at reduced resolution (every fifth row and column). Experiments show that this is sufficient for reliably detecting features on the order of 20 $\mathrm{cm}$ high. The local obstacle avoidance algorithm dynamically chooses which portion of the image should be processed, based on the current vehicle speed, stopping distance, and expected cycle time of the perception/planning/control loop. The Ranger algorithmmerges individual stereo-producedelevation maps to

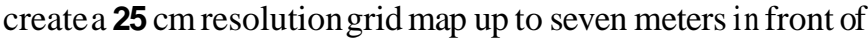
the rover. Map merging is necessary because the limited fields of view of the cameras do not allow a single image to view sufficient terrain.

Ranger works by projecting the rover's state (position, roll, pitch, yaw) as it travels along a path. The projection is based on a desired steering angle, the vehicle dynamics, and the underlying terrain. The vehicle's currentpose, itsdynamics and the steering angle are used to determine the position and yaw of the vehicle at the next time step. The height of the terrain under the wheels is then used to determine the roll and pitch of the vehicle at that point.

Once a projection of the vehicle along a path has been computed, the vehicle state at each point in time is evaluated. Four criteria are used to determine the "goodness" of a path: roll, pitches of the left and right body segments of Ratler, and number of known terrain points the vehicle crosses along the path. If any of the criteria exceed a given threshold of safety (such as excessive roll or pitch), the whole path is given a very low evaluation. Otherwise, the criteria are normalized to the range [0.1] and are combined using a linear weighted function. This determines the overall merit of choosing that steering angle for the rover. These evaluations are then combined with the user's preferences (Section 3) to determine the overall best command, which is then sent to the rover to be executed. The cycle time for this process is about 2 seconds, with the stereocomputations taking up about $75 \%$ of the time.

While the Ranger algorithm has worked well for high-speed navigation of Hum vees, it is not entirely well-suited to the much smaller, and slower, lunar rover.

As is often the case in robotics, the problems are mainly attributable to an abundance of noise: noise in the the stereoproduced terrain maps and noise in the dead-reckoning (particularly, the roll, pitch and yaw angles). The main effect of the noise was to make it difficult to cleanly merge terrain maps acquired from separate images, which is required by the Ranger algorithm since it uses only a small percentage of each image. Map 


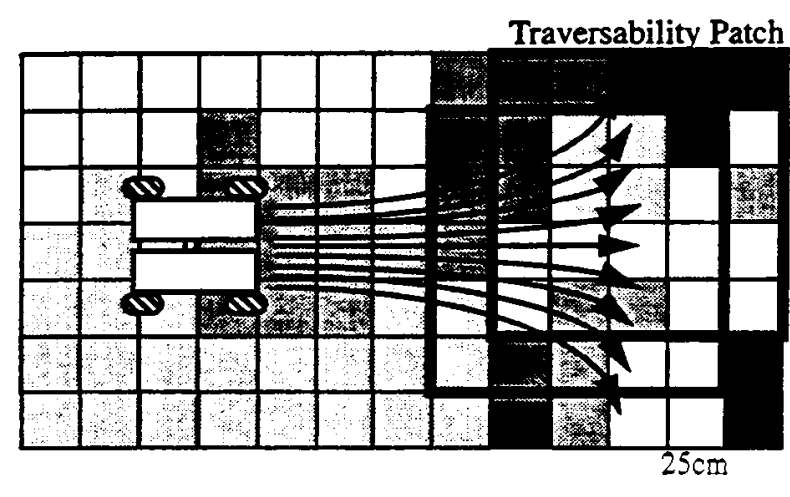

Figure 6: Local obstacle avoidance planner

merging often produced artifacts in the map, such as crevasses and ridges, which the rover would refuse to cross. This is less of an issue with the Humvees. since they can cross much taller obstacles. We tried several merging techniques in an attempt to minimize the artifacts, but none was robust enough to yield consistent driving results.

Another effect of noisy terrain data is that, because of the rover's relatively short wheelbase, small deviationsin perceived terrain elevation under the wheels produced relatively large changes in estimated roll and pitch. For example, a $20 \mathrm{~cm}$ "spike" in the terrain map (not uncommon) produces a $\mathbf{1 3}$ degree change in pitch, given a $90 \mathrm{~cm}$ wheelbase. Thus, it was often difficult to distinguish noise from steep bumps. This same problem also made it difficult to reliably determine whether high-centering might occur, since the clearance of the rover was not much more than the noise in the map. Finally, the Ranger algorithm presumed that the rover could track the path exactly, and not not account for uncertainty in the execution of commands or for uncertainty in the vehicle dynamics models used to project paths.

Toaddress these problems, we modified parts ofthe Ranger algorithm, creating a program called Morphin (a "power" Ranger). In contrast to the path-based approach of Ranger, Morphin is area-based: it analyzes patches of terrain to determine the traversability of each patch, and evaluates the traversability of a path by determining the set of patches it travels through. As such, it is actually more akin to Ranger's predecessor [24].

Each local terrain map. produced from a single stereo pair, is analyzed independently. The terrain is divided into overlapping patches, each $125 \mathrm{~cm}$ on a side, with patches offset by $25 \mathrm{~cm}$ from one another. Thus, each terrain point contributes to 25 patches (Figure 6).

To determine traversability, a plane is fit to each patch using least-squared error. To avoid redundant computation. statistics (e.g., sum of $\mathbf{X}$, sum of $X Y$ ) are collected for smaller 25 squared $\mathrm{cm}$ patches and then aggregated to determine the plane parametas for each 125 squared $\mathrm{cm}$ patch. The plane parameters are used in determining the vehicle roll and pitch (see below), and the residual to the plane fit is used to estimate the roughness of the terrain. Actually, two "roughness" measures are computed: one based on the residual in fitting the plane to the whole patch, and one based on the residual of each small $(25$ squared $\mathrm{cm}$ patch). The former indicates the roughness of the overall area, while the latter indicates if the patch is bumpy/spiky. Finally, the uncertainty in the evaluation is estimated in two ways: based on the total number of terrain points within the patch and the distributionof those points (essentially, the entropy of the spatial distribution).

Morphin then uses the Ranger projection algorithm to project the path of the rover over the terrain patches. If the center of the rover falls within the central 25 squared $\mathrm{cm}$ of a patch, the associated roughness and uncertainty measures for that patch are added to the overall path evaluation. The pitch of the vehicle is easily calculated as the slope of the line along the plane in the direction of the current heading (yaw). A similar calculation yields the vehicle roll. If there are overlapping patches from different images, only the one associated with the most recently acquired image is used (given the dead-reckoning uncertainty of the rover, we find this to be much more effective than trying to combine overlapping evaluations in some way).

Our navigation experiments (Section 6) have demonstrated the superiority of the Morphin algorithm for our rover. Morphin addresses the problem of noisy data by aggregatingindependent data points into an overall statistic, thus dramatically lessening the impact of any single point. While this aggregation can sometimes cause the rover to behave more conservatively than would otherwise be warranted, in our application it is better to be too conservative than to allow the rover to head into danger.

The remaining weakness of the algorithm is that the stereo vision often cannot craters, and reports them as unknown ares on the map. To handle such situations intelligently. Morphin needs to have a better understanding of spatial continuity: it needs to distinguish disjoint patches of truly unknown areas from contiguous unknown patches that may represent craters and other drop-offs.

\section{Field Trials}

To validate the safeguarded teleoperation approach, and gain insight into its strengths and weaknesses, we performed an extensive field trial. The test site (Figure 7) is an open field of area 2,000 square meters, bounded by a river and the Robotics Engineering Consortium building in Pittsburgh. The ground surface consists of soil, crumbled asphalt, loose gravel, scree, and some grassy vegetation. Obstacles to rover passage include soil mounds, depressions, cliffs at the river bank, building walls, metal pipes, cement blocks, railroad ties, trees, and bushes.

In previous outdoor experiments and field trials, the test method was to identify a goal (position and heading), and evaluate performance based on the accuracy with which the rover reached the goal. Unlike previous efforts, this field trial was formulated to test the performance of safeguarding given a careless and occasionally malicious driver. The trial called for the driver to explore the test site, at times covering ground systematically, at other times driving aimlessly, and at still other times driving maliciously, intentionally trying to drive into the river or at an obstacle. The performance criteria is the number of safeguarding failures.

On-board, we employed one 486 and one 386 processor. Offboard, we used a Sparc 10/30 (SuperSparc processor) with 32 MB memory for stereo, and a Sparc IPX (SunW 4/50 processor) with $48 \mathrm{MB}$ memory for obstacle avoidance planning.

We conducted the trial using a video tether, because the microwave video link performed poorly, probably because of the proximity to the river and interference from broadcasting by 


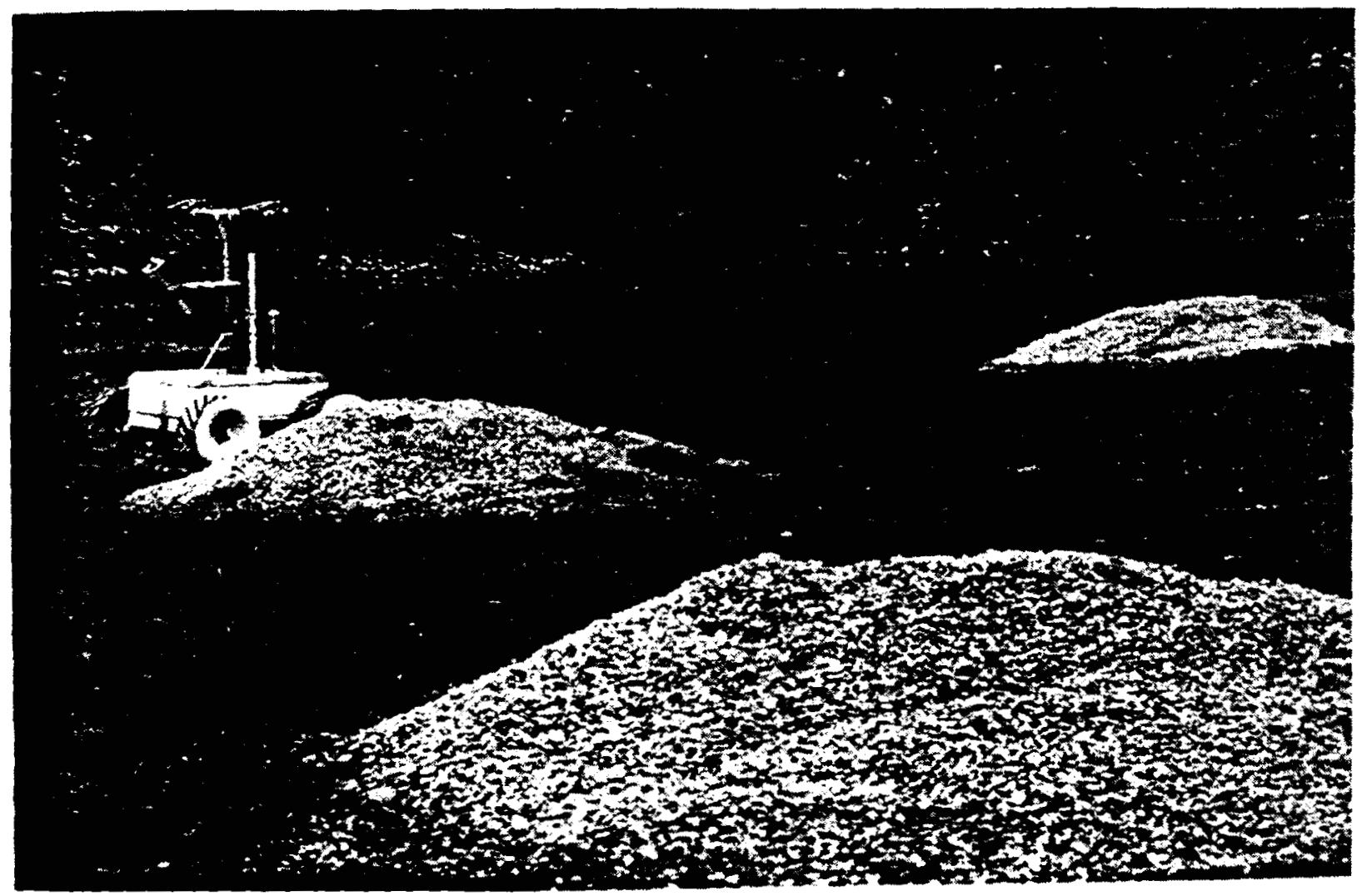

Figure 7: Test site

Traverse (meters), total

10,205

Traverse (meters), first day

1,163

Traverse (meters). second day

Traverse (meters), third day

Typical speed ( $\mathrm{cm} / \mathrm{sec})$

Operator interventionsper 100 obstacles (est)

Operator drive time percentage (est)

Number of stereo pairs matched (projected)

Overall cycle time (seconds), mean

Stereo cycle time (seconds), mean

Stereo cycle time (seconds), st. dev.

Obstacle avoidance cycle time (sec), mean

Obstacle avoidance cycle time (sec), st. dev.

Number of $3 \mathrm{D}$ pixels per cycle, mean

Number of $3 \mathrm{D}$ pixels per cycle, st. dev.

Projected number of $3 \mathrm{D}$ pixels processed
4,528

4.514

$15-20$

1

9,135

4.477

3.414

0.440

1.063

0.104

1,612

359

$3,279,465$
Figure 8: Field trial statistics

local businesses. This required tether management, but did not limit the scope of the trial.

Figure 8 summarizes the statistics of the field trial. The rover traveled over $10 \mathrm{~km}$ in one period of 3 days, during which the same problems arose again and again. In practioe, the rover could have covered greater distances, but given the repetitiveness of the problems, further testing would not be likely to cause new types of problems to appear.

One set of problems was due to obstacle detection. Stereo performed poorly at depressions, producing a significant number of unknown measurements, and a larger than usual number of outlier measurements. These problems accounted for virtually all of the operator interventions. Stereo performed with competence at discrete obstacles such as rocks and soil mounds, and functioned well despite varying lighting conditions

Another set of problems' involved system timing and synchronization. An example is spastic behavior after switching between safeguarded and pure teleoperation. Another example is unreasonably slow recovery from situations in which forward progress is blocked (the rover should back up or begin rotating, but does nothing for some time).

A third set of problems was caused by component limitations. Problems included communication dropouts, epoxy debonding, and electronics overheating. These are not fundamental,in the sense that buying higher-quality devices is the solution.

Acting maliciously, we attempted to drive the rover into hazardous situations. In one scenario, we drove as fast as possible at an obstacle, trying to $\mathrm{ram}$ it. The safeguarding system naturally resists operation \& ramming speed, because the rover rapidly outruns the known data, and Morphin treats unknown data as if it were a known obstacle. In another scenario. we aimed at a discrete obstacle. In these cases. Morphin prevented contact by planning and executing an alternative trajectory around the obstacle. In a third scenario, we aimed at an extended obstacle. In these cases, Morphin would begin evasive maneuvers as in the case of the discrete obstacle. However, no amount of looking left or right would reveal a traversablepath. So Morphin would halt the vehicle. Exception monitors then kicked in, causing the rover to back up and/or turn aggressivelyuntil it could find a new 
path. In summary, the safeguarding system performed competently at detecting and avoiding hazardous situations, even when drivers acted maliciously.

We observedinteresting behavior when traveling in areas with sharp, well-defined shadows. Under such conditions, the rover appears to pick either the shadowed or unshadowed region, and to avoid the shadow boundary. The reason for this unintended behavior is that the auto-iris lens adapts to either the brighter or the darker scene, whichever happens to occupy a larger portion of the image. After adaptation. the image portions with poor contrast begin to correlate less and less, causing the number of matches to fall, in tum causing the number of unknown areas to climb, causing the rover to steer away from that area.

We observed another in teresting behavior when driving down a dirt road. Vegetation on either side of the road caused those areas to appear as untraversable, producing the unintended behavior of remarkably robust road-following.

\section{Discussion}

In this paper, we have presented recent advances in developing and validating the safeguarded teleoperation approach to timedelayed remote driving. We presented results from experiments on untrained teleoperators with and without safeguarding. The results were inconclusive, but indicated that developing and evaluating the user interface raised a number of inter-related issues. We described three technical achievements in the safeguarding system: improving the accuracy of dead reckoning by a factor of 2 , speeding the controller up by a factor of 18 , and developing an area-based rather than a path-based obstacle avoidance planner in order to circumvent map merging problems. Finally, we discussed the $10 \mathrm{~km}$ field trial, which demonstrated the effectiveness of safeguarding, even with malicious drivers.

Future work will continue to push safeguarded teleoperation to new performance peaks. We will add a proximity sensor to detect the depressions and dropoffs that stereo does not see well enough. We anticipate that this will enable the system to traverse a wide variety of terrains without any form of intervention. We will mount all computing resources on-board, moving from Spares running SunOS to Pentiums running Linux. We expect that this will reduce cycle time by a factor of two or more. We will upgrade communication componentry, including a radio-frequency link for commands and data and a video link for camera imagery. Finally, we will achieve still higher levels of reliability and performance, continuing to refine the safeguarded teleoperation approach until time-delayed remote driving becomes routine, and we elevate our effonts to the next frontier: the Moon.

\section{Acknowledgments}

This research was partially sponsored by NASA under grants NAGW-1175 and NAGW-3863. We gratefully acknowledge significant contributions to this work by Ben R. Brown, Lonnie Chrisman, Diana Gant, Richard Goodwin, Martial Hebert, Lalitesh Katragadda Paul Klarer, Patrick Muir, Yoshiakazu Shinoda, Gregory Whelan, and Red Whittaker.

\section{References}

[1] A. Balazs, J. Biro, and S. Szalai. Transputer-based onboard computer. In Proc. Workshop on Computer Vision for Space Applications, pages 396-411, Antibes, France. September 1993.

[2] L. Boissierand L, Marechal. Rover demonstratorfor Moon exploration. Autonomous Robots, 2(4):353-362, December 1995 .

[3] R. Brooks, P. Maes, M. Mataric, and G. More. Lunar base constructionrobots. In Proc. IEEE Intl. Workshopon Intelligent Robots and Systems, pages 389-392, Tsuchiura, Japan, July 1990.

[4] R. Chatila, S. Lacroix, T. Simeon, and M. Herrb. Planetary exploration by a mobile robot: Mission teleprogramming and autonomous navigation. Autonomous Robots, 2(4):333-344, December 1995.

[5] Y. Fuke and E. Krotkov. Dead reckoning for a lunar rover on uneven terrain. In Proc. IEEE Intl. Conf. Robotics and Automation, Minneapolis, To appear, April 1996.

[6] J. Kay and C. Thorpe. STRIPE: Supervised telerobotics using incremental poly gonal-earth geometry. In Proc. Intl. Conf. Intelligent Autonomous Systems, Pittsburgh, February 1993.

[7] A. Kelly. An intelligentpredictive control approach to the high speed cross country autonomous navigation problem. Technical Report CMU-CS.TR-95-33, Carnegie Mellon University, 1995.

[8] E. Krotkov, J. Bares, L. Katragadda, R. Simmons, and R. Whittaker. Lunar rover technology demonstrations with Dante and Ratler. In Proc. Intl. Symp. Artificial Intelligence, Robotics. and Automationfor Space, Jet Propulsion Iaboratory, Pasadena, California, October 1994.

[9] E. Krotkov, M. Stereo perception and dead reckoning for a prototype lunar rover. Autonomous Robots, 2(4):313-331, December 1995.

[10] E. Krotkov and R. Simmons. Perception, planning. and control for autonomous walking with the Ambler planetary rover. Intl. J. Robotics Research, 15(2); ?, To appear, April 1996.

[11] M. Lamboley, C. Proy, L. Rastel, T. Trong, A. Zashchirinski. and S. Buslaiev. Marsokhod: Autonomous navigation tests on a Mars-like terrain. Autonomous Robots, 2(4):345352, December 1995.

[12] P. Lescoe, D. Lavery, and R. Bedard. Navigation of military and space unmanned ground vehicles in unstructured terrains. In Proc. Conf. on Military Robotic Applications, September 1991.

[13] S. Mathan, A. Hyndman, K. Fischer, J. Blatz, and D. Brams. Efficacy of a predictive display, steering device, and vehicle body representation in the operation of a lunar vehicle. In Human Factors in Computing Systems: CHI 96 Conference Companion, page ?, Vancouver, To appear. April 1996.

[14] L. Marthies. Stereo vision for planetary rovers: Stochastic modeling to near real-time implementation. Intl. J. Computer Vision, 8(1):71-91, July 1992.

[15] L. Matthies, E. Gat, R. Harrison, B. Wilcos, R. Volpe, and T. Litwin. Mess microrover navigation: Performance evaluation and enhancement. Autonomous Robots, 2(4):291312, December 1995. 
[16] R. Newman. Time lag considerations in operator control of lunar vehicles from Earth. In C. Cummings and H. Lawrence, editors, Technology of Lunar Exploration. Academic Press, 1962.

[17] R. Pagnot and P. Grandjean. Fast cross-country navigation on fair terrains. In Proc. IEEE Intl. Conf. Robotics and Automation, pages 2593-2598, Nagoya, Japan, May 1995.

[18] S. Parkes, A. Gillions, and S. Price. A processing architecture for space-based computer vision systems. In Proc. Workshop on Computer Vision for Space Applications, pages 322-334, Antibes, France. September 1993.

[19] C. Proy, B. Hotz, O. Faugeras, P. Gernesson, and M. Berhod. Onboard vision system for a mobile planetary exploration robot. In Proc. Workshop on Computer visionfor Space Applications, pages 2-12, Antibes, France, September 1993.

[20] D. Shirley and J. Matijevic. Mars Pathfinder microrover. Autonomous Robots, 2(4):283-290, December 1995.

[21] R. Simmons, E. Krotkov, L. Chrisman, F. Cozman, R. Goodwin, M. Hebert, G. Heredia, S. Koenig. F? Muir, Y. Shinoda, and W. Whittaker. Mixed-mode control of navigation for a lunar rover. In Proc. SSLPrinceton Space Manufacturing Conference, Princeton, New Jersey, May 1995.

[22] R. Simmons, E. Krotkov, L. Chrisman, F. Cozman, R. Goodwin, M. Hebert, L. Katragadda, S. Koenig, G. K.ishnaswamy, Y. Shinoda, W. Whittaker, and P.Klarer. Experience with rover navigation for lunar-like terrains. In Proc. Intl. Conf. Intelligent Robotos and Systems (IROS), pages $441-446$, Pittsburgh, Pennsylvania, Aug. 1995.

[23] R. Simmons, E. Krotkov, L. Katragadda, and M. Heat. Experience with rover navigation for lunar-like terrains. In Proc. Intl. Lunar Exploration Con., San Diego, California, November 1994.

[24] A. Stentz. The NAVLAB System forMobile Robot Navigation. PhD thesis, Carnegie Mellon University, 1989. 